\title{
MAPS PRESERVING THE LOCAL SPECTRUM OF THE SKEW JORDAN PRODUCT OF OPERATORS
}

\section{Abdelali Achchi, Mohammed Mabrouk and Rabi Marzouki}

Abstract. Let $\mathscr{H}$ and $\mathscr{K}$ be two infinite-dimensional complex Hilbert spaces, and fix two nonzero vectors $h_{0} \in \mathscr{H}$ and $k_{0} \in \mathscr{K}$. Let $\mathscr{L}(\mathscr{H})$ (resp. $\mathscr{L}(\mathscr{K})$ ) denote the algebra of all bounded linear operators on $\mathscr{H}$ (resp. on $\mathscr{K}$ ), and let $\mathscr{F}_{2}(\mathscr{K})$ be the set of all operators in $\mathscr{L}(\mathscr{K})$ of rank at most two. We show that a map $\varphi$ from $\mathscr{L}(\mathscr{H})$ into $\mathscr{L}(\mathscr{K})$ such that its range contains $\mathscr{F}_{2}(\mathscr{K})$ satisfies

$$
\sigma_{\varphi(T) \varphi(S)^{*}+\varphi(S)^{*} \varphi(T)}\left(k_{0}\right)=\sigma_{T S^{*}+S^{*} T}\left(h_{0}\right), \quad(T, S \in \mathscr{L}(\mathscr{H})),
$$

if and only if there exist a unitary operator $U$ from $\mathscr{H}$ into $\mathscr{K}$ and a scalar $\alpha \in \mathbb{C}$ such that $U h_{0}=\alpha k_{0}$ and $\varphi(T)=\lambda U T U^{*}$ for all $T \in \mathscr{L}(\mathscr{H})$, where $\lambda$ is a scalar of modulus 1 .

Mathematics subject classification (2010): Primary 47B49, Secondary 47A10, 47A11. Keywords and phrases: Nonlinear preservers, local spectrum, skew-Jordan product.

\section{REFERENCES}

[1] Z. Abdelali, A. ACHCHI, AND R. MARZOUKI, Maps preserving the local spectrum of skew-product of operators, Linear Algebra and its Applications 485 (2015), 58-71.

[2] P. AienA, Fredholm and local spectral theory, with applications to multipliers, Kluwer Academic Publishers, 2004.

[3] A. Bourhim AND M. MaBrouk, Maps preserving the local spectrum of jordan product of matrices, Linear Algebra and its Applications 484 (2015), 379-395.

[4] A. Bourhim AND M. Mabrouk, Jordan product and local spectrum preservers, Studia Math 234 (2016), 97-120.

[5] A. Bourhim AND J. MAShreghi, Maps preserving the local spectrum of product of operators, Glasgow Mathematical Journal 57 (2015), no. 03, 709-718.

[6] A. BOURHIM AND J. MASHREGHI, Maps preserving the local spectrum of triple product of operators, Linear and Multilinear Algebra 63 (2015), no. 4, 765-773.

[7] A. BouRhIM AND J. MASHREGHI, A survey on preservers of spectra and local spectra, CRM Proceedings and Lecture Notes: Invariant subspaces of the shift operator (RI. American Mathematical Society, Providence, ed.), (2015), 45-98.

[8] A. Bourhim AND J. MASHREGHi, Local spectral radius preservers, Integral Equations and Operator Theory 76 (2013), no. 1, 95-104.

[9] A. BOURHIM AND T. RANSFORD, Additive maps preserving local spectrum, Integral Equations and Operator Theory 55 (2006), no. 3, 377-385.

[10] J. BRAC̃̃Č AND V. MÜLLER, Local spectrum and local spectral radius of an operator at a fixed vector, Studia Math. 194 (2009), no. 2, 155-162.

[11] C. COSTARA, Automatic continuity for linear surjective mappings decreasing the local spectral radius at some fixed vector, Archiv der Mathematik 95 (2010), no. 6, 567-573.

[12] C. COSTARA, Linear maps preserving operators of local spectral radius zero, Integral Equations and Operator Theory 73 (2012), no. 1, 7-16.

[13] S. DU, J. HoU, AND Z. BAI, Nonlinear maps preserving similarity on $B(H)$, Linear Algebra and its Applications 422 (2007), 506-516. 
[14] K. B Laursen and M. M. Neumann, An introduction to local spectral theory, vol. 20, Clarendon Press, 2000. 